\title{
SOCIEDAD CIVIL Y CONCENTRACIÓN DE MEDIOS EN BRASIL
}

\section{Eula Dantas Taveira Cabral}

Trabaja en las areas de Investigación y de Comunicación de IBICT - Instituto Brasileño de Información en Ciencia y Tecnología del Ministerio de Ciencia y Tecnologia do Brasil.

PhD y Msc en Comunicación Social por la Universidade Metodista de São Paulo - UMESP. Email: eulacabral@gmail.com

\section{Adilson Vaz Cabral Filho}

Profesor del Curso de Comunicación Social y de los Programas de Estudios Pós-graduados en Política Social y de Posgrado en Medios y Vida Cuotidiana de la Universidade Federal Fluminense. Presidente da ULEPICC Brasil y vice-chair de la Sección de Comunicación Comunitaria de IAMCR desde 2012.

PhD y Msc en Comunicación Social por la Universidade Metodista de São Paulo - UMESP. Coordinador del grupo de investigación EMERGE - Centro de Pesquisas e Produção em Comunicação e Emergência. Email: acabral@comunicacao.pro.br

\section{Resumen}

El propósito de este artículo es comprender las formas de acción de la sociedad civil en el momento de la concentración de medios en Brasil. La investigación demuestra que la sociedad civil tiene una importancia considerable para cambiar la imagen actual de la concentración y de las irregularidades de los medios de comunicación de Brasil, basado en una investigación bibliográfica y documental, así como entrevistas con académicos y representantes de organizaciones sociales de la sociedad civil. Aunque se considere no actor / actriz de la regulamentación de los medios en Brasil, es a través de sus movilizaciones constantes al gobierno brasileño que las leyes se están construyendo en beneficio de la población brasileña y el temade la concentración de medios ha surgido en el país, siendo una de las cuestiones sensibles del segundo mandato de la presidenta Dilma Rousseff.

\section{Palabras clave}

Sociedad civil. Concentración de los medios de comunicación en Brasil. Economía política de la comunicación. Regulamentación de los medios en Brasil.

\section{CIVIL SOCIETY AND MEDIA CONCENTRATION IN BRAZIL}

\begin{abstract}
The purpose of this article is to understand ways of civil society action facing of media concentration in Brazil. Research shows that civil society has considerable importance to change the current picture of concentration and irregularities of the Brazilian media, based on bibliographical and documentary research as well as interviews with scholars and representatives of social organizations of civil society. Although considered a non-actor / actress of media regulation in Brazil, through its constant demonstrations to Brazilian government that laws are being built for the benefit of Brazilian population and the theme of media concentration has emerged in the country, being a sensitive issues for the second mandate of President Dilma Rousseff.
\end{abstract}

Keywords

Civil society. Media concentration in Brazil. Political economy of communication. Media regulamentation in Brazil. 


\title{
SOCIEDADE CIVIL E CONCENTRAÇÃO DA MÍDIA NO BRASIL
}

\begin{abstract}
Resumo
O objetivo deste artigo é compreender as formas de atuação da sociedade civil diante da concentração midiática no Brasil. A pesquisa mostra que a sociedade civil tem considerável importância para mudar o quadro atual de concentração e de irregularidades da mídia brasileira, com base em pesquisas bibliográfica e documental, bem como de entrevistas com representantes acadêmicos e de organizações sociais da sociedade civil. Apesar de considerada um não-ator/atriz da regulamentação da mídia no Brasil, é através de suas cobranças constantes ao governo brasileiro que leis vêm sendo construídas em prol da população brasileira e o tema concentração da mídia veio à tona no país, sendo um dos temas sensíveis do segundo mandato da presidente Dilma Rousseff.
\end{abstract}

\section{Palavras chave}

Sociedade civil., Concentração dos meios de Comunicação no Brasil. Concentração da mídia brasileira. Economia política da comunicação. Regulamentação da mídia no Brasil.

\section{INTRODUCCIÓN}

Los medios de comunicación tradicionales y del sector de las telecomunicaciones en Brasil se caracterizan por la concentración en unos pocos grupos. La realidad brasileña comprende detalles en el desarrollo del sistema de comunicaciones en y para el país, especialmente en relación con el fortalecimiento y el apoyo del sector privado.

Dada la concentración de grupos de medios de comunicación, su amplia expansión y su alcance en dimensiones territoriales, incluidas conexiones regionales y locales, la decisiva influencia que ejercen en la elaboración de las políticas en sus áreas de acción permite la comprensión de la concentración en el país como la más grande del mundo.

A pesar de los conglomerados de comunicaciones y telecomunicaciones privadas y muchos políticos en Brasil no teneren en cuenta la sociedad civil, su papel todavía se puede entender como fundamental para cambiar la imagen actual de la concentración y de las irregularidades de los medios de comunicación brasileños.

\section{METODOLOGÍA}

Para llevar a cabo esta investigación científica, se trabajó con la investigación bibliográfica y documental, entrevistas con representantes académicos y de organizaciones de la sociedad civil, como el FNDC, Comunicativistas y la ONGArtículo 19. Se cuestionó como se posicionan ante la concentración de los medios de comunicación de Brasil; si se puede cambiar; cómo el gobierno podría contribuir; si las normas y la regulación son caminos posibles; cuál es su 
papel; Se puede considerar como un de los actores de la regulación en Brasil y cómo podría intervenir.

\section{ESCENÁRIO MEDIÁTICO}

Brasil es uno de los principales países en América Latina y el séptimo lugar en la economía mundial. Su ámbito territorial es 8,515,767,049 km².Con más de 190 millones de habitantes, ocupa el quinto lugar entre los más poblados del mundo. Se divide en cinco regiones con 26 Estados, 1 Distrito Federal y 5.570 municipios.

En Brasil, la televisión abierta y la radio llegan a más del 90\% de los hogares. 96,9\% de los hogares brasileños tienen al menos un aparato de televisión en el país y el 83,4\% una radio (PNAD, 2013). TV por cable, teléfonos fijos y móviles e Internet se están convirtiendo en realidad para la mayoría de los brasileños. PNAD 2013 registró que el acceso a internet en los hogares alcanzó 85,6 millones de brasileños, o sea, 49,4\% de la población. Se ha verificado que 45,3\% de la población conéctasepor las microcomputadoras y un 4,1\% de las personas a través de otros dispositivos tales como teléfonos móviles, tablet o televisión.

De acuerdo con la Investigación Brasileña de Medios 2015: los hábitos de consumo de medios de comunicación por la población brasileña, el 95\% de los encuestados asisten la televisión (73\% ven la televisión todos los días), el 55\% escucha la radio (con el 30\% escucha todos los días) y el $48 \%$ accesan laInternet. $21 \%$ lee periódicos y $13 \%$ revistas. También se registra que el 26\% de los hogares brasileños son atendidos por el servicio pago de televisión, 23\% para antena parabólica y el 72\% tienen acceso a la emisión de TV.

En Brasil, a diferencia de otros países, el área de la radiodifusión (radio y televisión) se separa de las telecomunicaciones. Hay un modelo de funcionamiento de la radio y televisión por parte de grupos privados brasileños liderados por políticos, familias e iglesias. Por otra parte, no hay regulamentación y regulación de los medios de comunicación.

Brasil tiene la mayor concentración de los medios en el planeta. En el área de la radiodifusión, cinco conglomerados nacionales de medios de comunicación privados y cinco grupos regionales llegan a casi $100 \%$ del territorio brasileño. Los nacionales alcanzan 5.570 municipios. 
Rede Globo está en 98,6\%, es decir, 5.490 ciudades de Brasil, alcanzando el 96,7\% de los hogares con televisión. Segundo es el SBT (Sistema Brasileño de Televisión) con 85.7\%, ganando 4.772 municipios y el 92,2\% de los hogares con televisión. En tercer lugar viene Rede Record con 79,3\% o 4.417 municipios, alcanzando 90,2\% de los hogares con televisión. En cuarto lugar, Rede Bandeirantes con $64,1 \%$ en 3.569 municipios y el $87,6 \%$ de los hogares con televisión. Y en quinto lugar, en la investigación de Mídia Dados 2015, aparece la Rede TV con $56,7 \%$, llegando a 3.157 municipios y el 76,5\% de los hogares con televisión.

En el caso de los grupos regionales, se evidencian: RedeBrasil Sul (en el Sur del país) alcance en todos los hogares de Río Grande do Sul y Santa Catarina; Organizaciones Jaime Câmara (en el Centro Oeste); Red Amazónica de Radio y Televisión que opera en cinco de los siete estados de la región Norte; Grupo Zahran, en los estados de Mato Grosso y Mato Grosso do Sul; y Grupo Verdes Mares (en Nordeste).

La concentración de medios en manos de unos pocos conglomerados en Brasil es tomada como ilegal. Esto es posible porque no hay cumplimiento de la ley, no se cumple el plazo para el cambio legalde los propietarios y no existen restricciones a la formación de redes nacionales y regionales. Estas infracciones se pueden observar en la legislación brasileña, especialmente en consideración al capítulo V de la Constitución Federal vigente (1988).

Además de la concentración en el área de la radiodifusión por propietarios brasileños, en la de Telecomunicaciones el agravante es que todos los propietarios son extranjeros y alcanzan casi 100\% del territorio nacional. Seis grupos son: Telefónica / Vivo / GVT (en telefonía fija, móvil, banda ancha y TV de pago); Oi (en telefonía fija, móvil, banda ancha y TV de pago); América Móvil BR (formado por Claro, Embratel y Net, opera en telefonía fija, móvil, banda ancha y TV de pago); TIM (en el móvil y banda ancha); Nextel (operadora deSME- trunking comunicación por radio); y SKY (en banda ancha y TV de pago).

La industria de las telecomunicaciones en Brasil en el primer trimestre 2015 estuve representada por la prestación de servicios a 375 millones de suscriptores, un aumento de 1,2\% respecto al trimestre de 2014. Es decir, 45 millones con telefonía fija; 283,4 millones de teléfonos móviles; 19,8 millones con la televisión de pago; 24,4 millones con la banda ancha fija; y 2,5 millones de dólares con el SME, el Trunking.

Frente a un panorama interesante de los medios para los grupos de radiodifusión y telecomunicaciones, se forman oligopolios. Sin embargo, la concentración de los medios de 
comunicación puede restringir el mercado, evitando la democraciaen los medios de comunicación. Corresponde al gobierno federal regulamentar y regular el mercado. Los involucrados en el proceso de regulamentación de los medios (gobierno, empresas y sociedad civil) tienen que tomar y cumplir su función.

Según Venicio Lima (2011), el principal actor de regulamentación de los medios en Brasil es el Estado, o sea, el Poder Ejecutivo, y la Constitución de 1988 establece que la concesión y renovación de la radiodifusión deben ser compartidas con el Parlamento. Sin embargo, es importante señalar que los parlamentarios brasileños son los responsables de la renovación de los canales y al mismo tiempo propietarios. Algo que hace caso omiso de la Constitución de 1988 en su Artículo V, artículo 54, que registra que los diputados y senadores tienen prohibido firmar o mantener un contrato con empresas concesionarias de servicio público y no podrá también aceptar o ejercer cargos, función o empleo remunerado en las estaciones.

El segundo actor del proceso, detectado por LIMA (2011, p.33), se compone de grupos empresariales privados representados por entidades que actúan como actores políticos. Además del sector de la radiodifusión se incluyen las compañías telefónicas que operan actualmente en el país, en el sistema de telefonía móvil y residencial, Internet de banda ancha y televisión por cable; y la industria electrónica que fabrica equipos y tiene "interés directo en soluciones tecnológicas que se adoptarán para la implementación de nuevas tecnologías, especialmente las derivadas de la radio y la televisión de exploración".

Cabe destacar también la sociedad civil que, según Venicio Lima (2011, p.33) es un "noactor" que trabaja sus posiciones, "pero no son capaces de ejercer una influencia significativa", porque, para el investigador, lo que hay "una negociación entre el gobierno y algunos grupos privados". Pero a pesar de Lima (2011) ver la sociedad civil no como actor, es importante señalar que en Brasil muchos problemas en este sector surgen gracias a sus esfuerzos. Por lo tanto, su papel es crucial para cambiar la imagen actual de la concentración y de irregularidades de los medios de comunicación brasileños.

Ejemplo de acción de la sociedad civil es lo que sucedió en 2009, cuando el gobierno federal, en busca de la regulamentación de los medios, ha celebrado en Brasilia, la primera Conferencia Nacional de Comunicación, con asistenciade más de 1.600 delegados. De las entidades del sector privado, solamente asistieron ABRA (Asociación Brasileña de los Radiodifusores) y Telebrasil (del sector de las telecomunicaciones). El evento, a pesar de no 
contar con apoyo de los grupos de medios de comunicación, resultó en casi 700 propuestas com objetivo de ayudar al gobierno en la regulación de los medios de comunicación en Brasil.

Además de la Conferencia, en el gobierno de Lula, en julio de 2010, el Presidente hizo un decreto que creó una Comisión Interministerial para preparar estudios y presentar propuestas para la revisión del marco regulador de los servicios de radiodifusión y de telecomunicaciones. En 2014, la Presidente de Brasil, Dilma Rousseff, ha prometido que en su segundo mandato (comenzado en 2015) defienderia la regulación de los medios en Brasil. Sin embargo, ya que es un tema "sensible", sobre todo en relación con el derecho a la información y la democratización de los medios de comunicación, encuentran resistencia del Congreso Nacional y "ofrecido" por los conglomerados de medios a la población como la censura de la libertad de expresión.

\section{POSICIONAMENTO DE LA SOCIEDAD CIVIL A RESPECTO DE LA CONCENTRACIÓN MEDIÁTICA BRASILEÑA}

La participación de la Sociedad Civil (SC) en procesos como los de las cumbres y conferencias multilaterales de las Naciones Unidas consolidase y da frutos en la formulación de propuestas, reivindicaciones y protestas, así como el monitoreo de las políticas públicasel monitoreo de las políticas públicas. Si desde la década de 1970, con el desarrollo del movimiento ambientalista, sus miembros comenzaron a tejer la idea de pensar globalmente y actuar localmente, a finales de la década de 1990, con el fortalecimiento de la sociedad civil en las redes globales, esta perspectiva ha llegado a construirse con base en las ideas del pensamiento global y la acción global.

Sin embargo, el crecimiento de las articulaciones en muchos niveles diferentes y la necesidad de las personas de diferentes comunidades, desde el nivel más simple a niveles más complejos, desde consensos en torno a mejores prácticas y estrategias, ha refuerzado demandas relacionadas con el pensar y actuar global, pero con los pies en el local. Un local que genera movimientosen escala nacional y que recibeal revés los frutos de las articulaciones regionales y globales generados desde ahí, como en debates en torno a la comunicación como el interés público.

La SC organizada tiene un papel clave en la conformación de las políticas públicas, para que sean reclamadas y afirmadas en su hacer cuotidiano. Su lugar es mucho menos para afirmar 
la composición de una estructura tripartite, en la cual ella se identifica desde la comprensión estrecha del tercer sector, pero, sin ignorar la existencia de momentos de necesarias concertaciones junto con el Estado y el mercado, afirmar su autonomía desde diferentes actividades, que les permiten dar identidad junto a la población en general.

También hay que entender la complejidad de estas atribuiciones, en la medida en que el Estado también se constituye por actores hegemónicos de la sociedad civil y sus fuerzas de apoyo, afirmando y prevaleciéndo se del poder regulador,que crea, evalua y realiza varios ajustes. La apropiación social aparece no sólo como estrategia de uso de las TIC disponibles o a reivindicarse, sino como un eje central de la articulación de los actores, en el contexto de las organizaciones de la sociedad civil, para el desarrollo de nuevas regulaciones.

En el campo de la comunicación, se debe entender de cuales formas se hacen posible la promoción del interés público, como se refieren a la comunicación con el sistema actual. Las iniciativas que fomentan la articulación de redes y el aprendizaje de una manera compartida contribuyen a proporcionar modelos de referencia para una democratización de la comunicación que se afirma como derecho humano desde el propio poder-hacer comunicacional.

Mientras que el gobierno federal no se ponede modo claro, la sociedad civil, desde 2013, busca firmas de la población para que la Iniciativa de Projecto de Ley de Iniciativa Popular de Comunicación Social Electrónica (PLIP), conocida como la Ley de Medios Democrática, entre en la agenda del Parlamiento brasileño. También funciona con diputados federales y senadores con propuestas para hacer de la democratización de las comunicaciones una realidad en el país.

Al consultar las instituciones y los círculos académicos que conforman la sociedad civil y luchan en favor de la Democratización de la Comunicación sobre como se posicionan frente a la concentración de los medios, los grupos Comunicativistas, FNDC y el Artículo 19 y dos representantes de la academia respondieron a las preguntas.

Comunicativistas es el Colectivo por una Comunicación Libre y Democrática, formada por activistas de diversos sectores. El FNDC es el Foro Nacional para la Democratización de la Comunicación opera en la planificación, la movilización, la formulación y el desarrollo de las medidas jurídicas y políticas para promover la democracia en la comunicación. El Artículo 19, en su turno, trabaja para que cada persona se exprese libremente, acceder a la información y disfrutar de la libertad de prensa. 
Es importante destacar que, aunque no se considere uno de los actores de regulación de los medios en Brasil, la SC está se involucrando suficiente en los debates sobre la concentración de los medios de comunicación brasileños.

De acuerdo con la representante de Comunicativistas, Cláudia Abreu (2015), hay una participación de la SC en las "luchas y movilizaciones en torno a las luchas por la democratización de la comunicación. La concentración de los medios de comunicación es cada vez mayor y con dañosa los trabajadores y la sociedad del sector en general". Por lo tanto, es necesario que la SC estea "expandiendo el número de actores, que prohíbe la propiedad cruzada mediante la imposición de los límites necesarios para evitar la concentración del sector. Apoyamos el PLIP, un proyecto que tiene como objetivo democratizar el sector en las líneas de los marcos legales vigentes".

Para el representante de FNDC, Orlando Guilhon (2015), FNDC, desde 1991, "lleva esta batalla contra el monopolio / oligopolio en los medios de comunicación privados / comerciales brasileños". Suportan "proyectos que regulan la regionalización dela producción periodística y cultural en la radiodifusión", como las "campañas nacionales para aprobar una Ley de Medios Democrática que regule la prohibición del monopolio / oligopolio en los medios de comunicación nacionales, peticiones, debates y seminarios sobre universidades, sindicatos y organizaciones sociales".

GUILHON (2015) también ha explicado que FNDC produce materiales como folletos, videos, audios, etc., entrevistas y materiales para los medios alternativos, comunitarios, libres y públicos y hace actos y manifestaciones públicas en calles y plazas, campañas en las redes sociales, negociaciones y presión sobre los poderes públicos, acciones legales en el MPF y en la Corte Suprema. "De todos modos, hemos utilizado todas las formas posibles de lucha y movilización para tratar de avanzar en esta lucha en Brasil".

Sobre la posibilidad de cambiar el escenario actual, GUILHON (2015) llama la atención sobre el hecho de que la SC debe combinar diversas estrategias de lucha, cómo recolectar 500.000 firmas para el Proyectode Ley de Medios Democráticosa seranalisado en el Congreso.

La abogada Karina Quintanilha (2015), representante de La ONG Artículo 19, explica que el grupo "cree que la concentración de los medios de comunicación es una gran amenaza para la libertad de expresión en Brasil" y se han movilizado para que pongan fin en esta situación. Llama la atención sobre la necesidad de debates sobre la legislación de los medios. "Entendemos que 
esta situación implica discutir medios y leyes para evitar un monopolio en la radiodifusión". Además, "asegurar la expansión y sostenibilidad de los medios públicos y comunitarios, así como presionar para la existencia de los organismos reguladores de comunicación verdaderamente independientesy imparciales."

En cuanto a la concentración de las telecomunicaciones, el investigador senior del Centro de Políticas, Derecho, Economía y Tecnologias de Comunicación y profesor de la Universidad de Brasilia, Murilo César Ramos (2015), llama la atención sobre el hecho de que "se trata de un mercado que, como de radiodifusión, es oligopolio - y se podría argumentar que es un oligopolio natural, dados los altos costos de entrada, en términos de inversión en infraestructura física y recursos de tecnología". En relación a "la competencia entre los grupos existentes, es un mercado más equilibrado. El desequilibrio se puede ver en la televisión de pago, en el que el grupo Claro / Embratel / Net se concentra más de 50\% del mercado". Sin embargo, el investigador cree que el escenario va a cambiar en los próximos años "con la creciente competencia de las llamadas empresas over-the-top (OTTs), es decir, aquellas como Netflix y similares, Facebook, Google / YouTube, Apple, Amazon, con su oferta vídeo a la carta y firmas más baratas a través de Internet".

Analizando el mercado de la radiodifusión y las telecomunicaciones, Murilo César Ramos (2015) señala que existe desequilibrio económico, "el mercado de la radiodifusión, cuyos ingresos brutos anuales poco superan $\mathrm{R} \$ 20$ mil millones anuales, mientras que el mercado de las telecomunicaciones ha tenido, en 2014, los ingresos brutos totales de más de R\$ 200 mil millones".

Según el profesor de la Escuela de Comunicación de la Universidad Federal de Río de Janeiro, vicepresidente de ULEPICC-Br, Marcos Dantas (2015), la estructura del mercado de las comunicaciones es crítica. En lo que se refiere a las telecomunicaciones, señala que "el discurso de la concentración es un discurso liberal, constantemente desmentido por la realidad. (...) En gran parte del país, están presentes uno o dos operadores".

En cuanto a la radiodifusión, Marcos Dantas (2015) registra que "la escasez de frecuencias de aire también obligaba la limitacióndel número de actores y favorecia la lógica de concentración del capital. Hoy en día, esta realidad está cambiando". Para el investigador, "en términos prácticos, nosotros y mi organización (ULEPICC-Br) trabajamos en la construcción de un marco legal para abrir espacio para la entrada de nuevos actores no comprometidos con el uso 
de la lógica". Es decir, "sería ocupado por los productores y difusores interesados en proponer y apoyar a los discursos y imágenes alternativas a la cultura de mercado".

Pero sin la participación del Gobierno Federal, la SC considera que no es posible cambiar el escenario actual. Para Orlando Guilhon (2015), el Gobierno Federal "está de manos atadas, por no ser exactamente una regulación del capítulo 220 de la Constitución Federal, que prohíbe explícitamente el monopolio y oligopolio en los medios de comunicación en el país". Pero al mismo tiempo, señala que "el Gobierno debe tener la audacia de llamar para sí la responsabilidad de hacer este debate [reglamentos] de la forma más amplia, transparente y republicana", y sobre todo "la participación de todos los sectores de la sociedad, llevando adelante las resoluciones de la I Conferencia Nacional de Comunicación".

Cláudia Abreu (2015) llama la atención para el hecho de que el Gobierno debería "poner este tema en la agenda, distribuyendo mejor las inversiones de publicidad y formular políticas con la participación de la sociedad". El investigador Marcos Dantas (2015) sostiene que se debe también "usar algunos mecanismos de la legislación vigente para fomentar la diversidad en los medios de comunicación" y / o "podría asumir el liderazgo de la presentación y defensa de un proyecto de ley, de acuerdo con la Constitución brasileña, que fomente la diversidad en los medios de comunicación".

Para QUINTANILHA (2015), el Ejecutivo, el Legislativo y el Judiciario deben tomar medidas "para prevenir y para obstaculizar la concentración en la radiodifusión para asegurar pluralismo y diversidad". El legislativo "debe discutir con la sociedad un proyecto de ley que regule de forma efectiva los artículos de la Constitución Federal que se ocupan de la Comunicación Social”. Considera que "es un deber del Congreso Nacional crear parámetros claros en la ley para las garantías expresas en este capítulo, eso incluye la prohibición del monopolio en los medios y la garantía de sustentabilidadde los medios comunitarios y públicos".

En lo referente al Ejecutivo, Karina Quintanilha (2015) explica que, con basis en la ley actual, "cabe aumentar el monitoreo de prácticas ilegales de emisoras que abusan de las concesiones (arriendando la programación, con transferencias de concesiones, duplicación de la antena, etc)". Por otra parte, utilizar el CADE - un organismo de control económico brasileño "para sancionar prácticas del monopolio y de propiedad cruzada". En su turno, el Judiciario, "se abstene de aplicar el Derecho Penal para criminalizar las emisoras, a através del uso del principio de la insignificância y del derecho internacional cuando tratar de las radios comunitarias”. 
Para Murilo César Ramos (2015), “el gobierno de la presidenta Dilma Rousseff empezó, con timidez, una propuesta llamada regulación económica de los medios de comunicación”.El afirma que "el gobierno podría llamar para si mismo el proceso de reglamentación, al menos en partes vitales del Capítulo de Comunicación Social de la Constitución Federal”. Pero frente a un escenario con problemas en el Congreso, "nada indica que será hecho de pronto, incluso teniendo en cuenta las condiciones políticas difíciles que afectan al año 2015, sobre todo en relación con el Congreso Nacional". Para el investigador, "ni Luiz Inácio Lula da Silva, ni Dilma Rousseff estuvieron cerca, en estos casi 13 años de gobiernos del PT, para cumplir sus promesas de una mayor democracia para los medios".

Las reglas y la regulación de la legislación sobre las comunicaciones brasileñas se consideran posibles maneras de poner fin a la concentración de los medios de comunicación en Brasil. Para Cláudia Abreu (2015), se necesita sólo que se regule lo que ya se ha señalado en la Constitución. Orlando Guilhon (2015) añade: "La simple aplicación mas dura de algunas disposiciones legales por parte del Ministerio de las Comunicaciones, tanto en la política de concesión de otorgas, como en la prohibición de propiedadde canales a políticos podría haber daño fatal a este poder monopólico de los médios”.

Karina Quintanilla (2015) explica que la regulación se hace en conformidad con las normas internacionales de libertad de expresión, tendrá que "el potencial para distribuir uniformemente el espacio en el espectro electromagnético entre los sectores público, comunitario y privado, diluyendo el poder y la rentabilidad de ciertos grupos políticos y econômicos". Se llama la atención el hecho de que "un proceso de regulación debe ser creado para poner en práctica un plan para las frecuencias atribuidas a la radiodifusión, con el fin de ser promovidos al máximo, como modo de garantizar la diversidad". Sin embargo, "el proceso debe ser abierto y con la participación de todos y debe ser supervisado por un organismo que está protegido contra la interferencia política y comercial".

Murilo César Ramos (2015) señala que hay tres formas de regulación y de control posibles: la presión de la sociedad; iniciativa del poder Ejecutivo y del Legislativo. "La sociedad civil progresista tiene más posibilidades de intervención en el proceso de reglamentación y reguladores de las telecomunicaciones de lo que nunca tuvieron en la radiodifusión”. En el caso de la radiodifusión, “aunque 20 veces menos en peso económico, centra mucho más político de 
peso que las telecomunicaciones, que representan hoy, como lo han hecho desde la disputa por la Ley n. 4117, mayor desafío político para la sociedad civil brasileña”, dijo RAMOS (2015).

Para el representante del artículo 19, Karina Quintanilha (2015), "le a la sociedad civil empoderarse de las discusiones presentadas por las normas internacionales y las experiencias de los países vecinos", que exigen "que el Estado cumpla con su deber de garantizar positivamente el derecho a la comunicación a través de la legislación, el fomento de la actividad de comunicación y la supervisión de este sector”. Según el investigador Murilo César Ramos (2015), el papel de la sociedad civil es "continuar con la formulación de propuestas, en el mundo académico y más allá, los sindicatos, las más variadas organizaciones populares, y con estas propuestas, siempre presionar los Poderes Ejecutivo y Legislativo". Y añade, "no se puede abandonar este papel, ya que es, y seguirá siendo el corte protagonista en la lucha por la democratización de los medios de comunicación en el país”.

\section{CONCLUSIONES}

Así pues, parece que la sociedad civil busca posicionarse y requiere más acción por parte del gobierno federal, debilitado frente a los desacuerdos ocurridos entre Ejecutivo y Legislativo y la gran presión ejercida por algunos sectores políticos y económicos, incluidos los medios de comunicación.

Debemos transformar el panorama de los medios sobre una base democrática, que comprende como esenciales el combate y superación de la concentración de los medios de comunicación en el país. Desafío en torno del cual la sociedad civil ha jugado un papel clave en Brasil. 


\section{BIBLIOGRAFÍA}

ABREU, Cláudia. Resposta dos Comunicativistas sobre a Concentração da Mídia no Brasil. Email enviado à pesquisadora Eula D.T.Cabral no dia 18 de junho de 2015.

BRASIL. Presidência da República. Secretaria de Comunicação Social. Pesquisa brasileira de mídia 2015: hábitos de consumo de mídia pela população brasileira. Brasília: Secom, 2014. Disponível em: <http://www.secom.gov.br/atuacao/pesquisa/lista-de-pesquisas-quantitativas-equalitativas-de-contratos-atuais/pesquisa-brasileira-de-midia-pbm-2015.pdf >. Acesso em: 15 dez.2015.

CABRAL, Eula D.T. Concentração da Mídia no Brasil: Estudo dos Conglomerados de Radiodifusão e Telecomunicações. Rio de Janeiro: Pesquisa de Pós-Doutorado. Universidade do Estado do Rio de Janeiro, 2015.

CABRAL FILHO, Adilson V. Políticas de Comunicação Comunitária: elementos para um modelo de análise. In: Anais do XXXVI Congresso Brasileiro de Ciências da Comunicação. Manaus: Intercom, 2013.

A emergência nos processos comunicacionais: um paradigma entre a política e a expressão popular. In: Anais do VII Congreso Internacional de Comunicación Lusófona. Santiago de Compostela: LUSOCOM, 2006.

CABRAL, Eula Dantas Taveira. Mídia da sociedade civil, direitos à comunicação e a transição para o digital no Brasil: estabelecendo uma estrutura analítica para uma perspectiva comparada internacional. In Revista Brasileira de Políticas da Comunicação, v. 1, p. 1-14, 2011. Disponível em: <http://rbpc.lapcom.unb.br/pdf/RBPC-1-1.pdf>. Acesso em: 15 dez.2015.

DANTAS, Marcos. Concentração da Mídia no Brasil. Email enviado à pesquisadora Eula D.T.Cabral no dia 23 de junho de 2015.

GUILHON, Orlando. Resposta do FNDC sobre a Concentração da Mídia no Brasil. Email enviado à pesquisadora Eula D.T.Cabral no dia 19 de junho de 2015.

LIMA, Venício Artur de. Regulação das Comunicações: História, Poder e Direitos. São Paulo: Paulus, 2011.

PNAD 2013. IBGE. Disponível em: <http://ftp.ibge.gov.br/Trabalho_e_Rendimento/Pesquisa_ Nacional_por_Amostra_de_Domicilios_anual/2013/Sintese_Indicadores/sintese_pnad2013.pdf $>$. Acesso em: 15 dez.2015.

QUINTANILHA, Karina. Resposta do Artigo 19 sobre a Concentração da Mídia no Brasil. Email enviado à pesquisadora Eula D.T.Cabral no dia 19 de junho de 2015.

RAMOS, Murilo César. Concentração da Mídia no Brasil. Email enviado à pesquisadora Eula D.T.Cabral no dia 30 de junho de 2015. 